\title{
Pancreatitis in children: diagnosis and etiology in 57 patients
}

\author{
J. M. Van Camp, T. Z. Polley, and A. G. Coran \\ Section of Pediatric Surgery, C.S. Mott Children's Hospital, and the University of Michigan Medical Center, Ann Arbor, MI 48109-0245, USA
}

Accepted 20 January 1994

\begin{abstract}
Pancreatitis in children is uncommon, but can be the cause of significant morbidity. We undertook this study to review recent changes in the management of pediatric pancreatitis. Between 1974 and 1992, 57 cases of pancreatitis in children up to 19 years of age were treated at our institution. The etiologies were quite diverse and included idiopathic, traumatic, drug-related, biliary, congenital, and alcoholic causes. The diagnosis of pancreatitis was made from the clinical presentation and laboratory values in most cases. Serum amylase was elevated in the majority of children. Further diagnostic evaluations included abdominal ultrasound, computed tomography, and endoscopic retrograde cholangiopancreatography. Pseudocyst development was the most common complication, especially in those cases associated with trauma. In contrast to adults with pancreatitis, who usually respond to nonoperative therapy, only 32 of the 57 children responded to nonoperative treatment. The most common long-term morbidity was recurrent pancreatitis; however, the majority of patients suffered no long-term sequelae of their pancreatitis or its treatment.
\end{abstract}

Key words: Pancreatitis - Childhood - Pediatric - Review

\section{Introduction}

Although rare in children, disorders of the pancreas may lead to significant morbidity and mortality, including retardation of normal growth and development. Pancreatitis has been found to be the most common pancreatic disorder in children in recent reviews, such as those of Synn et al. [8] and Vane et al. [10], which included congenital mal-

Correspondence to: J. M. Van Camp, c/o A. G. Coran, L2110 MCHC, Ann Arbor, MI 48109-0245, USA formations, endocrine disorders, and neoplasms. The most recent large series reported was that of Zeigler et al., who published a review of pancreatitis in 49 children in 1987 [16]. In 1986, Beshlian and Ryan [3] reported a series of 21 teenagers with pancreatitis. Little et al. [5] focused on chronic pancreatitis beginning in childhood, while Weizman and Durie [14] concentrated on acute pancreatitis in children in their series. The diagnosis and management of childhood pancreatitis has changed significantly in the last 5 years with more frequent use of endoscopic retrograde cholangiopancreatography (ERCP).

We have treated 57 children with pancreatitis during the last 18 years, including both acute and chronic cases. We have reviewed this series in order to evaluate changes in the etiology, diagnosis, and management over the past 2 decades.

\section{Materials and methods}

The records of all children with pancreatitis up to the age of 19 years treated at the University of Michigan C.S. Mott Children's Hospital between 1974 and 1992 were reviewed. The diagnosis of pancreatitis was based on the following data: a history of abdominal pain with either elevated serum or urinary amylase and no other determined cause of the pain. Seven children without elevated amylase levels were included on the basis of operative or autopsy findings consistent with pancreatitis.

\section{Results}

Fifty-seven patients met the criteria used for this review. There were 26 males and 31 females; the ages ranged from 21 months to 19 years (mean 12 years). Pancreatitis was the primary admitting diagnosis in 38 cases; the remaining children developed pancreatitis as a complicating factor in the management of hematologic disorders $(n=6)$, multiple trauma $(n=5)$, diabetic ketoacidosis $(n=2)$, cystic fibrosis $(\mathrm{n}=1)$, pseudotumor cerebri $(\mathrm{n}=1)$, lung transplantation $(n=1)$, rhabdomyosarcoma $(n=1)$, and tetrology of Fallot $(n=1)$. 


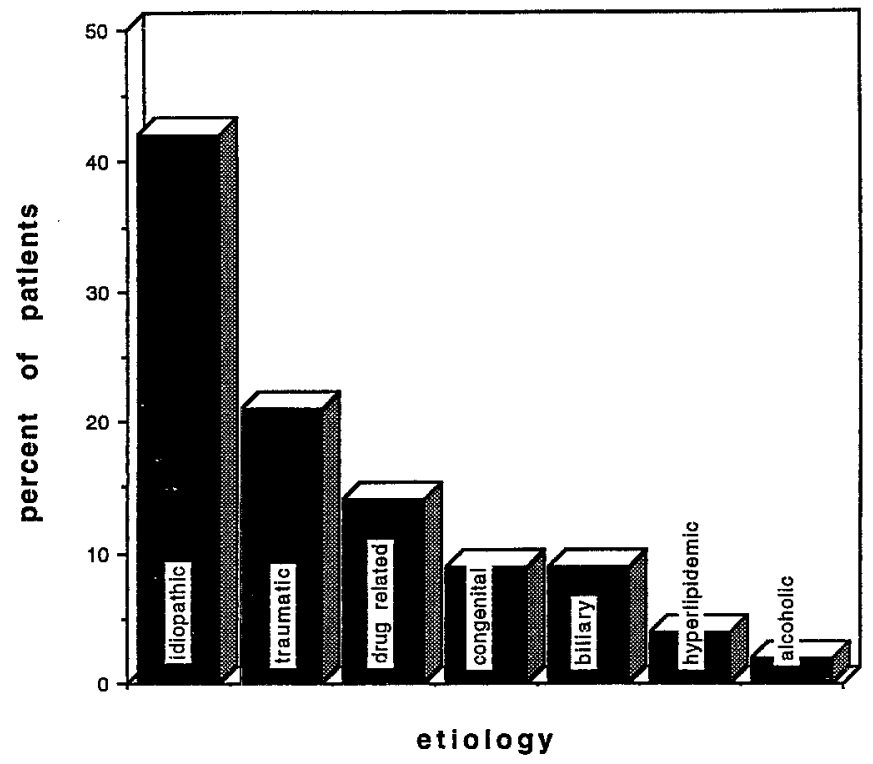

Fig. 1. Etiology of pancreatitis

No specific cause of the pancreatitis could be determined in 24 (42\%) of these children (Fig. 1), and these 24 have been labeled "idiopathic." Four had a family history of at least one other family member with pancreatitis, but there was not enough information to assign the diagnosis of hereditary pancreatitis. One was a 23 -month-old boy with severe hemorrhagic pancreatitis in whom child abuse was suspected but no further evidence of abuse was found.

Twelve children $(21 \%)$ developed pancreatitis as the result of trauma; 3 of these had injuries secondary to child abuse. Bicycle and motor-vehicle accidents were also common causes of traumatic pancreatitis. Drug therapy such as L-asparaginase, vincristine, or high-dose steroids was also a common cause. Pancreatitis occurred in 5 patients with congenital abnormalities of the biliary tract, including stenosis of the sphincter of Oddi and the presence of choledochal cysts. One child had pancreas divisum with stenosis of the entrance of the duct of Santorini into the duodenum and of the sphincter of Oddi. Five patients (9\%) developed pancreatitis secondary to choledocholithiasis, and 1 of these also had congenital stenosis of the sphincter of Oddi. Two children (4\%) had pancreatitis secondary to hyperlipidemia and 1 adolescent $(2 \%)$ developed alcoholic pancreatitis.

Fifty-four patients had documented abdominal pain at the time of diagnosis and 46 presented with nausea or vomiting. Other less common but specifically documented symptoms were fever $(n=9)$, diarrhea $(n=7)$, dehydration $(n=4)$, and jaundice $(n=3)$. Laboratory values at the time of diagnosis included a normal white blood count $\left(\right.$ WBC $=3000-10000$ cells $\left./ \mathrm{mm}^{3}\right)$ in 29 of the 51 children with a documented WBC. Eleven patients had mild WBC elevations (10000-15000 cells $/ \mathrm{mm}^{3}$ ) and only 2 had WBC greater than 20000 cells $/ \mathrm{mm}^{3}$. Elevation of serum amylase (>100 IU/l) was one of the criteria for inclusion in the series, and was found in 49 children. One of the remaining 8 children had elevated urinary amylase only, and the remaining 7 had morphologic or histologic evidence of pancreatitis found at operation or autopsy. Elevated serum

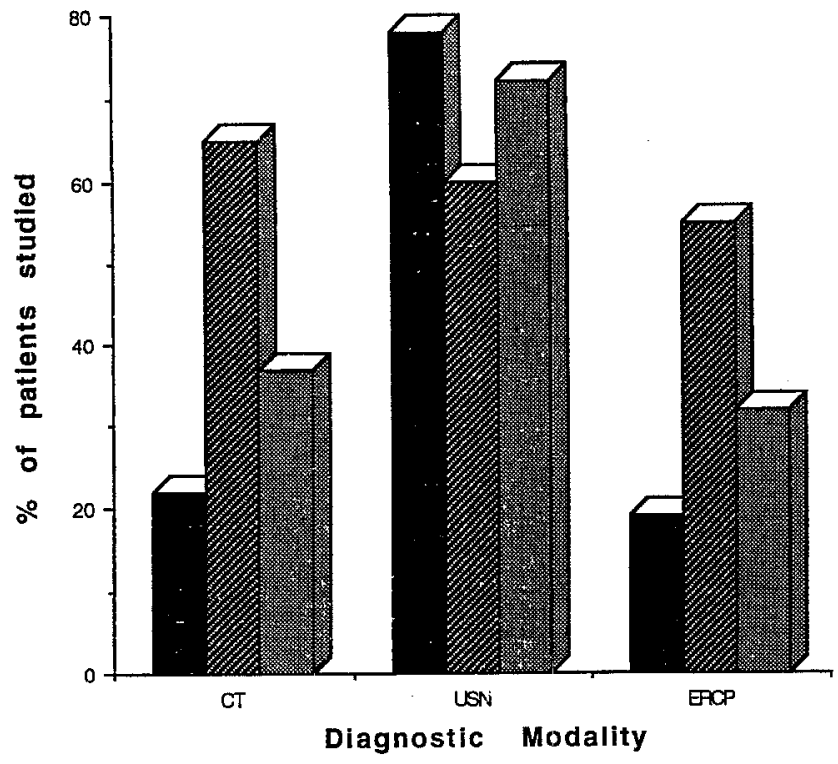

Fig. 2. Diagnostic studies $(\mathrm{CT}=$ computed tomography, USN $=$ ultrasonography, $\mathrm{ERCP}=$ endoscopic retrograde cholangiopancreatog-

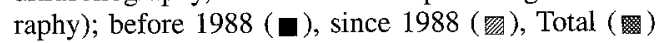

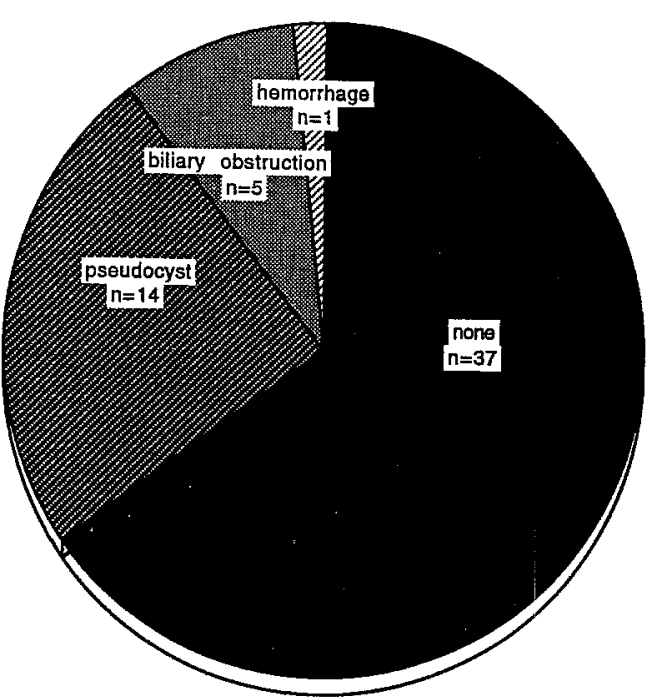

Fig. 3. Complications of pancreatitis

amylase levels ranged from 115 to 4640 IU/1 (average $881 \mathrm{IU} / \mathrm{l})$. Total bilirubin levels were less than $1.0 \mathrm{mg} / \mathrm{dl}$ in 28 of the 38 children in which they were documented. Only 3 children had total bilirubin levels greater than $3.0 \mathrm{mg} / \mathrm{dl}$.

The most common diagnostic modality used specifically to evaluate pancreatitis was ultrasound (US): 41 children underwent abdominal US (Fig. 2). Fifteen of these were read as normal; only 2 of these patients were found to have abnormal computerized tomography (CT) scans at the same admission. One of the abnormal CT scans showed an abnormally small pancreas, and one described a $1-\mathrm{cm}$ fluid collection in the head of the pancreas. Twenty-one patients underwent abdominal CT, only 3 of which were read as normal. ERCP was used in $17(30 \%)$ of the patients, however, $56 \%$ of the children admitted since 1988 underwent ERCP. 


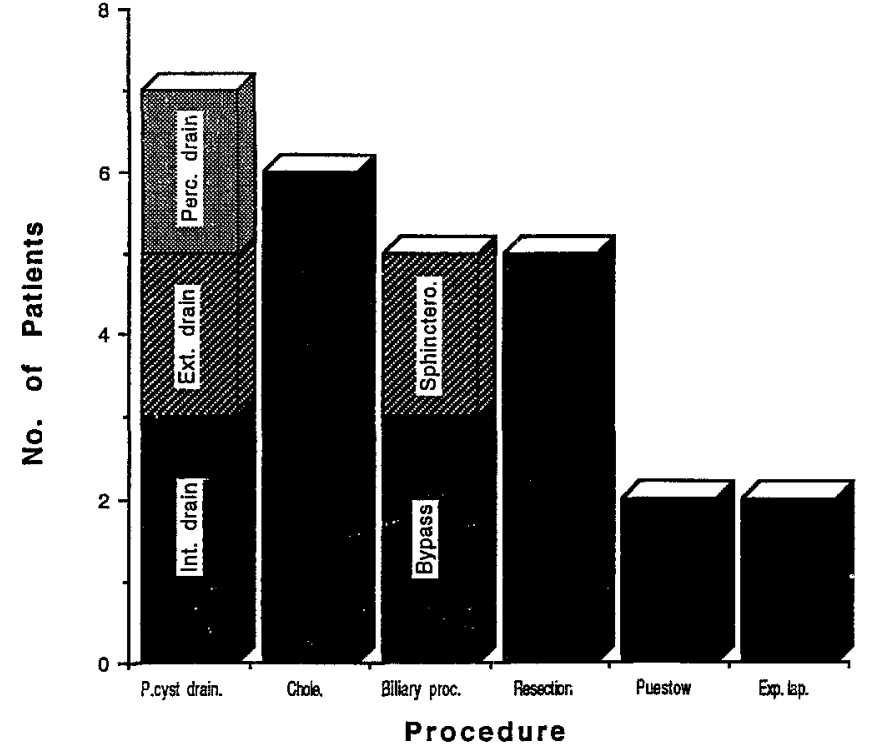

Fig. 4. Surgical management $(\mathrm{P}$. cyst. drain. = pseudocyst drainage, chole $=$ cholecystectomy, proc. $=$ procedure, Exp. lap. $=$ exploratory laparotomy, Int. drain = internal drainage, Ext. drain $=$ external drainage, Perc. drain $=$ percutaneous drainage, Sphinctero. $=$ sphincterotomy

The majority of patients treated for pancreatitis did not develop any complications (Fig. 3). The most common complication was the formation of pseudocysts, which occurred in 14 cases. Pseudocysts occurred in 50\% of children who presented with traumatic pancreatitis. Five children developed evidence of biliary obstruction secondary to acute or chronic pancreatitis. One developed bleeding into a pseudocyst, which required embolization by angiography.

Thirty-one children were treated nonoperatively, which included bowel rest, IV fluids, and variable use of nasogastric suction (Fig. 4); 19 received supplemental parenteral nutrition. Twenty-seven underwent some sort of operative procedure. All patients diagnosed with gallstone pancreatitis underwent cholecystectomy with intraoperative cholangiography or common bile duct exploration. Five patients underwent a partial pancreatectomy. Twelve of the 14 children who developed pancreatic pseudocysts required some type of surgical procedure; 2 underwent distal pancreatectomy, 2 had cyst-enteric drainage, 2 had external drainage, 1 had a Puestow procedure, and 2 underwent exploratory laparotomy. Three of these patients underwent simple percutaneous drainage of their pseudocysts, 1 of whom had a residual pseudocyst that eventually resolved spontaneously.

Two children died: a girl involved in a motor vehicle accident who died of a closed head injury, and a boy with leukemia who died of sepsis. For the 36 who were admitted primarily for pancreatitis, the average length of hospital stay was 13 days (range 2-61). Follow-up was obtained on 50 children (2-80 months); 28 of these have had no known recurrence or further problems. Six children had recurrent pancreatitis, 3 had exocrine deficiency, 2 had residual pseudocysts (both resolved spontaneously during outpatient follow-up within 6 months), 2 continued to complain of

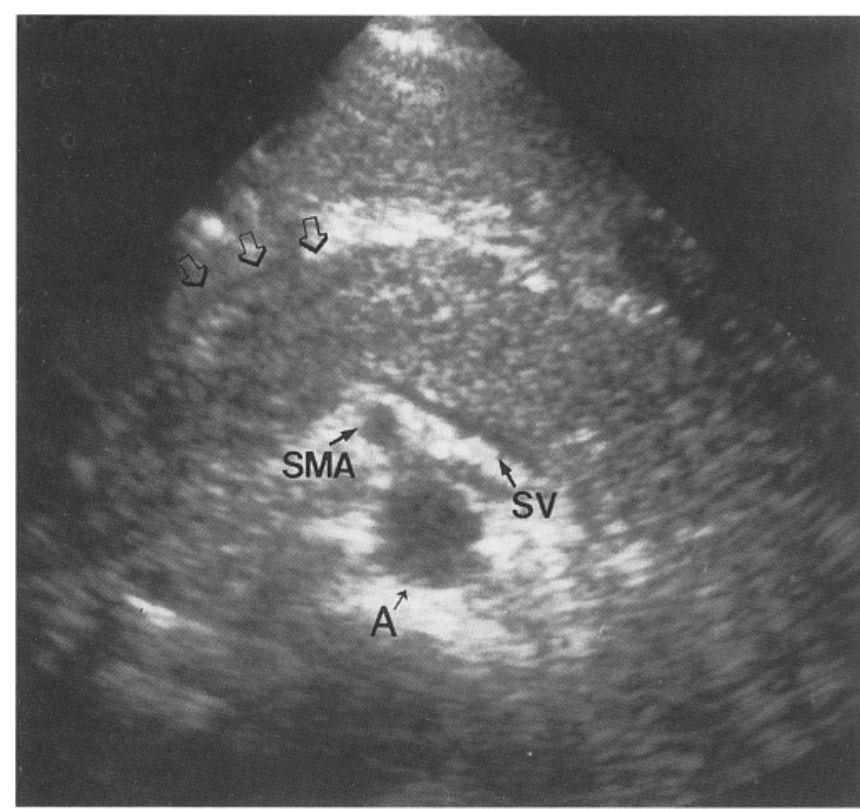

Fig. 5. US scan of a 15-year-old boy with biliary obstruction secondary to pancreatitis shows diffusely enlarged pancreas with no focal lesions. Open arrows, head of pancreas; SMA, superior mesenteric artery, $A$, aorta; $S V$, splenic vein

abdominal pain, and 1 required home parenteral nutrition for several weeks. The remaining 8 children received treatment for diseases that may or may not have been related to their pancreatitis. There was no significant difference between the rate of recurrent pancreatitis among children who underwent operation and those who did not.

\section{Discussion}

\section{Etiology}

Several aspects of pancreatitis in children differ from adult pancreatitis. Whereas biliary tract disease and alcohol abuse are the most common causes of pancreatitis in the adult, they are rare in children [8]. The causes in the pediatric population include trauma, drug therapy, congenital anomalies, hyperlipidemia, as well as choledocholithiasis and alcoholism [12, 14]. Other reported causes include malnutrition and viral, bacterial, and parasitic infection [12].

One of the most common causes of pancreatitis in children is trauma: injury to the pancreas occurs in up to $10 \%$ of cases of blunt abdominal trauma [2]. Fracture usually occurs in the body of the pancreas since this portion is relatively fixed over the spine, predisposing it to injury [11]. Previous reports have stated that the incidence of trauma-induced pancreatitis in children ranges from $19 \%$ to $33 \%[8,10,11]$. Child abuse is a major cause of trauma, occurring in $25 \%$ of trauma victims and up to $33 \%$ of cases of trauma-induced pancreatitis [11]. Child abuse should be considered as a possible etiology of pancreatitis in all children, but especially in younger children [16]. Another frequent cause of pancreatic trauma is bicycle handlebar injury. Up to $42 \%$ of the pediatric pancreatic trauma re- 

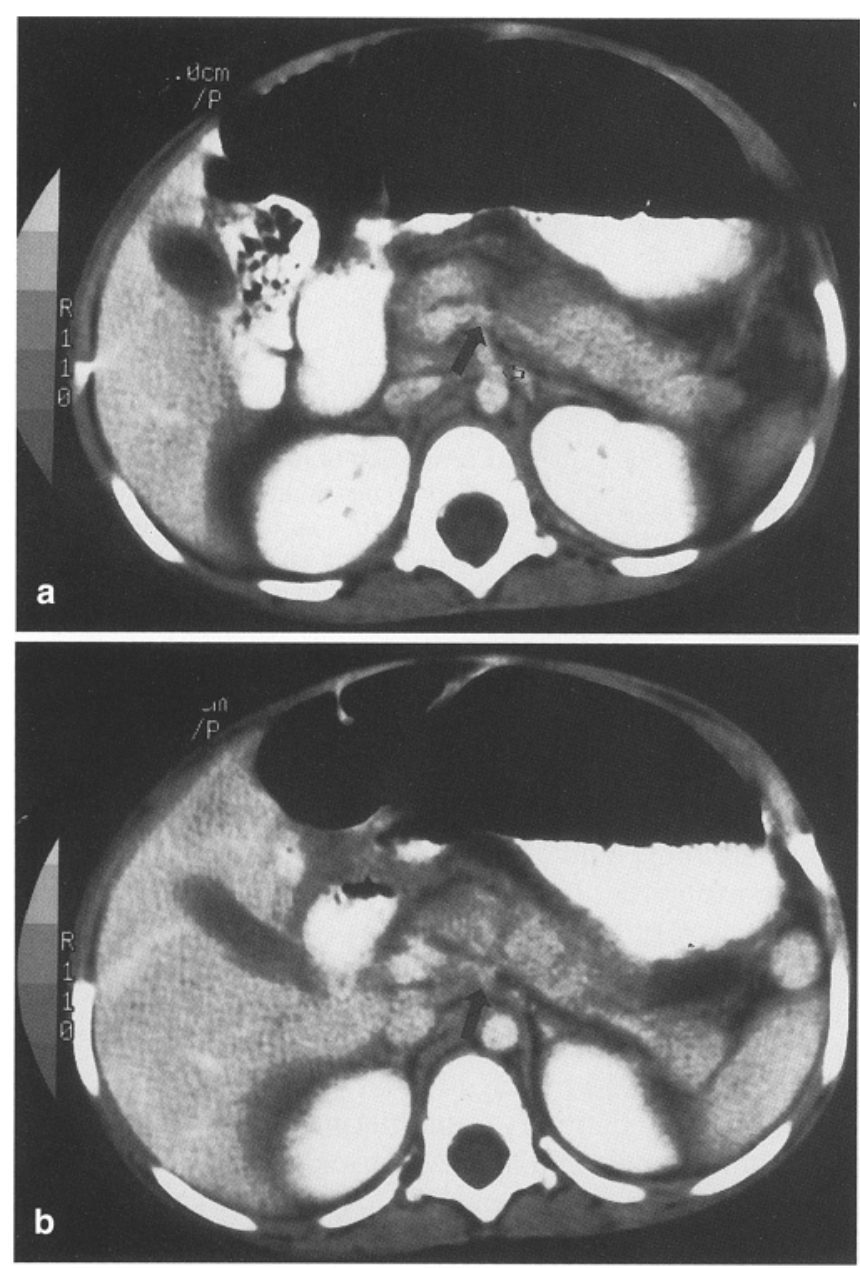

Fig. 6. A, B Abdominal CT of a 21-month-old boy 2 days after a motor vehicle accident. Pancreatic transection (large arrow) seen just to left of superior mesenteric artery (open arrow)

ported is bicycle-related [2]. Other causes of traumatic pancreatitis include motor-vehicle accidents, sports injuries, and penetrating trauma.

Therapeutic drug-induced pancreatitis was found in 8 of our patients. The chemotherapeutic agents L-asparaginase and vincristine and corticosteroids were the main causes. Other drugs that can cause pancreatitis in children include azathioprine, hydrochlorothiazide, and sulfonamides [3].

Congenital abnormalities of the pancreaticobiliary system were found in 5 of the patients in this series. Only 1 had a pancreas divisum, despite its presence in $10 \%$ of the Western population [13]. This is consistent with the theory that in order to cause disease, pancreas divisum must be accompanied by an additional factor such as papillary stenosis [13], as occurred in our patient. Choledochal cyst is also a cause of pancreatitis, although this is an uncommon presentation [3]. The risk factors for teenagers developing gallstone pancreatitis are similar to those of adults: female sex, obesity, family history, and pregnancy [3].

The most common etiology in this review was idiopathic $(n=24)$. This is slightly higher than that in other reports in the literature, which range from $6 \%$ to $33 \%[8$, 16]. Although it is rare in children, the most common cause of recurrent pancreatitis in the pediatric age group is hereditary pancreatitis [4]. There are two criteria for the diagnosis of hereditary pancreatitis: (1) pancreatitis must be present in three or more members of the same family; and (2) episodes of abdominal pain must be traced to childhood without other etiologic factors [4].

\section{Diagnosis}

The diagnosis of pancreatitis can be made with reasonable certainty on the basis of clinical, radiographic, and laboratory findings [6]. An overwhelming majority of our patients complained of abdominal pain, mostly described as diffuse or epigastric. However, abdominal pain may be difficult to assess in very young children or children involved in multiple trauma with possible head or extremity injuries. Nausea and vomiting was also found commonly in our review.

Janowitz considered serum amylase to be the most important diagnostic aid in determining pancreatic injury [12]. Although serum amylase may be normal in pancreatitis [7], up to $95 \%$ of cases of acute pancreatitis have elevated amylase levels [11]. It should be noted, however, that serum amylase may not rise until $12 \mathrm{~h}$ after the acute event [11]. WBC, lactate dehydrogenase, and total bilirubin levels were also found to be normal or only slightly elevated in the large majority of cases. Extreme elevation of these values should lead one to suspect serious complications of pancreatitis or other illnesses.

Abdominal US has been shown to have $80 \%$ accuracy in the evaluation of pancreatitis, usually demonstrating decreased echogenicity of the pancreas [8]. US is a noninvasive imaging modality that allows not only for diagnosis of the disease, but for following its course and detecting complications [7]. Because of its accuracy, noninvasiveness, speed, portability, and relative inexpensiveness, US should be performed in any case of suspected pancreatitis or unexplained abdominal pain [14].

$\mathrm{CT}$ has been shown to be the most valuable single imaging modality in children with suspected abdominal trauma $[10,11]$. It can be used not only to evaluate the pancreas, but also to evaluate the liver, spleen, gastrointestinal tract, spine, or other organs that may be involved in abdominal trauma. Therefore, CT should be considered in patients who present with traumatic pancreatitis but adds little to abdominal US when used for more isolated causes of pancreatitis.

ERCP is emerging as the most useful tool in the diagnosis and management of chronic pancreatitis in children [15]. It has a morbidity of $2 \%$ in children, consisting mainly of mild pancreatitis $[1,15]$. Relative contraindications to ERCP include acute pancreatitis and the presence of a pancreatic pseudocyst that is not being staged for operative drainage. Small size is only a relative contraindication, since cannulation has been successful in infants [1]. ERCP should be considered in the evaluation of idiopathic, nonresolving, or recurrent pancreatitis, and in all children with pseudocyst prior to operation [1]. 

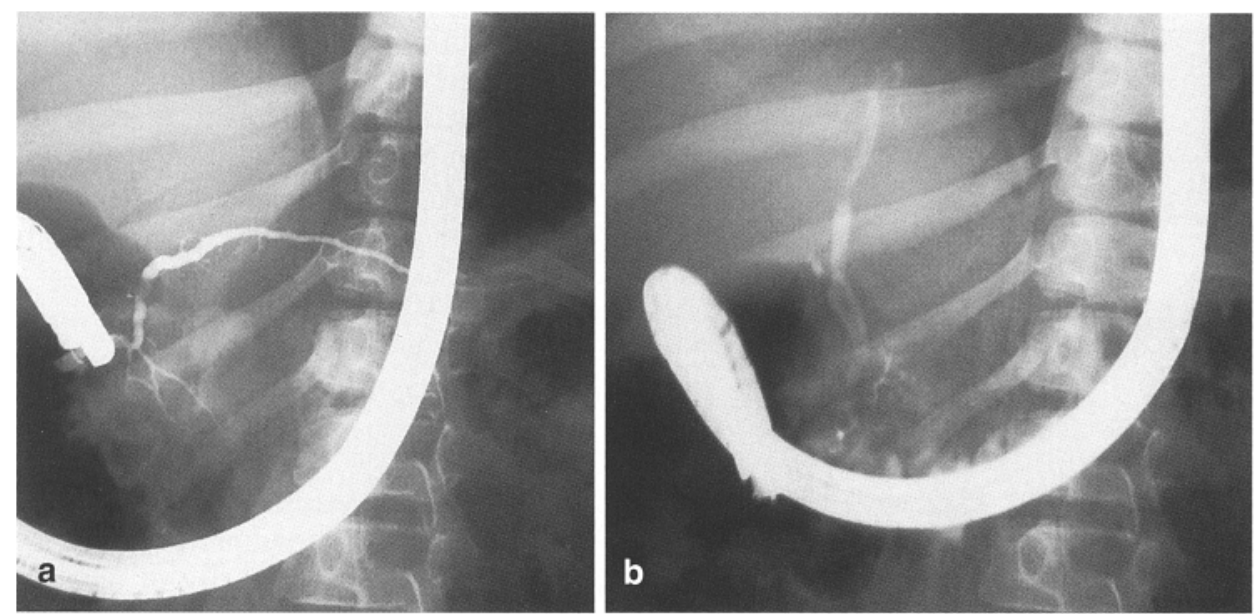

Fig. 7.A, B ERCP of a 10-year-old boy with history of recurrent pancreatitis. Cannulation of minor pancreatic papilla demonstrates dominant dorsal pancreatic duct $(D)$. Cannulation of the major papilla reveals small accessory duct $(E)$, consistent with pancreas divisum

\section{Complications}

The most common complication of childhood pancreatitis is pseudocyst formation $(10 \%-25 \%)$ [12, 14]. Pseudocysts occur most frequently in association with traumatic pancreatitis. Most childhood pseudocysts are acute and thinwalled and share no connection with the pancreatic duct. Pseudocysts in children are usually acutely symptomatic and require earlier treatment than those in adults [12]. Biliary obstruction secondary to acute or chronic pancreatitis is rare in childhood, and obstructive jaundice secondary to biliary stricture is an uncommon manifestation of childhood pancreatitis [15]. However, 5 patients in this series presented with obstructive jaundice secondary to pancreatitis. Two of these children were treated conservatively with resolution of their symptoms, and $3 \mathrm{had}$ bypass procedures.

\section{Treatment}

The treatment of pancreatitis consists of bowel rest and IV fluids with or without nasogastric suction; $30 \%$ to $76 \%$ of cases of pancreatitis in children can be treated conservatively $[3,8,16]$. This is in contrast to a large series of adults with pancreatitis, in whom $73 \%$ were treated nonoperatively [3].

The most common operation performed in our series was pseudocyst drainage. Results with percutaneous drainage have been reported to be better in children than in adults, probably due to the higher percentage of patients with the absence of primary pancreatic pathology [2]. However, among the patients drained percutaneously in this series, 2 required a further operation, 1 had a residual pseudocyst that resolved spontaneously, 1 had no recurrent problems, and 1 was lost to follow-up. Vane et al. also reported that two of three of their attempts at percutaneous drainage failed [10].

The second most common operation was cholecystectomy with intraoperative cholangiogram or common bile duct exploration. Although only $14 \%$ of children with choledocholithiasis develop recurrent pancreatitis while waiting for operation, as compared to $37 \%$ of adults, cho- lecystectomy during the initial hospitalization is recommended for all patients with gallstone pancreatitis [16].

Debate continues over the choice of surgical procedure for biliary obstruction. Choices include sphincteroplasty, sphincterotomy, and/or biliary bypass with choledochoduodenostomy or choledochojejunostomy [15]. A reported complication of these procedures is cholangitis [15], however, none of the patients in this series have developed cholangitis since their bypass surgery. Good results with sphincteroplasty for stenosis of the sphincter of Oddi have also been achieved by Tagge et al. [9] and O'Neil [17]. If only mild pancreatitis is found during an exploratory laparotomy for "acute abdomen," Ranson recommends that no further operative procedure (including placing drains) is appropriate [6].

\section{Outcome}

The mortality in pediatric pancreatitis varies greatly $(0 \%-$ $78 \%$ ) [16]. The average length of hospital stay for children treated primarily for pancreatitis in our series was 13 days. The most frequent long-term complication was recurrent pancreatitis, which occurred in 6 children, 4 of whom were in the idiopathic group. This high rate of recurrent pancreatitis in children diagnosed with idiopathic pancreatitis has also been noted in the literature [14]. In patients with recurrent pancreatitis metabolic (i.e., hyperthyroidism, hyperlipidemia, or cystic fibrosis), structural, or hereditary causes should be sought. If no metabolic cause is found, ERCP should be performed to rule out a structural cause [14].

Exocrine deficiency may also result from pancreatitis. Malabsorption has been reported in 5\%-45\% of adults as a complication of pancreatitis [5]. Two patients in our series were diabetic at the time they developed pancreatitis. This agrees with the known increase in the incidence of pancreatitis in diabetics $[1,5]$. Another long-term complication of childhood pancreatitis is persistent abdominal pain, which has been reported in $6 \%$ of children in one review [8] and occurred in $4 \%$ of our patients, both of whom had idiopathic pancreatitis. 
Although the majority of children with pancreatitis respond to nonoperative therapy, a high percentage require operation when compared to adults. The morbidity and mortality of pancreatitis in childhood is minimal.

\section{References}

1. Allendorph M, Werlin SL, Geenen JE, et al (1987) Endoscopic retrograde cholangiopancreatography in children. J Pediatr 110 : $206-211$

2. Bass J, DiLorenzo M, Desjardins JG (1988) Blunt pancreatic injuries in children: the role of percutaneous external drainage in the treatment of pancreatic pseudocysts. J Pediatr Surg 23: 721-724

3. Beshlian K, Ryan JA (1986) Pancreatitis in teenagers. Am J Surg 152: $133-138$

4. Franken EA, Chiu LC, Smith WL, et al (1984) Hereditary pancreatitis in children. Ann Radiol 27: 130-137

5. Little JM, Tait N, Richardson A, et al (1992) Chronic pancreatitis beginning in childhood and adolescence. Arch Surg 127: 90-92

6. Ranson JH (1990) The role of surgery in the management of acute pancreatitis. Ann Surg 211: 382-391

7. Rosenberg HK, Ortega W (1987) Hemorrhagic pancreatitis in a young child following valproic acid therapy. Clin Pediatr 26: 98-101
8. Synn AY, Mulvihill SJ, Fonkasrud EW (1988) Surgical disorders of the pancreas in infancy and childhood. Am J Surg 156: $201-205$

9. Tagge EP, Smith SD, Raschbaum GR, et al (1991) Pancreatic ductal abnormalities in children. Surgery 10: 709-717

10. Vane DW, Grosfeld JL, West KW, et al (1989) Pancreatic disorders in infancy and childhood. J Pediatr Surg 24: 771-775

11. Vasundhara T, Patel AS, Aumndson GM (1990) Pancreatic fracture secondary to child abuse: the role of computed tomography in its diagnosis. Clin Pediatr 29: 667-668

12. Warner RL, Biemann Othersen H, Smith CD (1989) Traumatic pancreatitis and pseudocyst in children: current management. J Trauma 29: 597-601

13. Warshaw AL, Simeone JF, Shapiro RH, et al (1990) Evaluation and treatment of the dominant dorsal duct syndrome (pancreas divisum redefined). Am J Surg 159:59-66

14. Weizman Z, Durie PR (1988) Acute pancreatitis in childhood. J Pediatr 113: 24-29

15. Wheatley MJ, Coran AG (1988) Obstructive jaundice secondary to chronic pancreatitis in children: report of two cases and review of the literature. Surgery 104: 863-869

16. Ziegler DW, Long JA, Philippart AI, et al (1988) Pancreatitis in childhood. Ann Surg 207: 257-261 